\title{
The Set-theoretic Multiverse
}

\section{as a Mathematical Plenitudinous Platonism Viewpoint ${ }^{\dagger}$}

\author{
Sakaé FuCHINO*
}

\begin{abstract}
We address aspects of mathematical Platonism and examine the possibility of Platonism viewpoint in mathematics in wake of recent devolopments in set theory.
\end{abstract}

Key words: philosophy of set theory, mathematical Platonism, set-theoretic multiverse

\section{Platonisum in mathematics}

In a popular description, mathematical Platonism in philosophy is explained as "the form of realism that suggests that mathematical entities are abstract, have no spatiotemporal or causal properties, and are eternal and unchanging" ([16]).

Kurt Gödel once described mathematical Platonism as:

"the view that mathematics describes a non-sensual reality, which exists independently both of the acts and [of] the dispositions of the human mind and is only perceived, and probably perceived very incompletely, by the human mind" ([9]).

Because of this and other remarks, as well as what he expressed in his [8] and elsewhere in his mathematical oeuvre, Gödel is considered to be a champion of mathematical Platonism. This, however, is quite misleading, since his "Platonism", and the Platonism shared by most set-theorists, is different kind of Platonism from that held by the majority of mathematical Platonists who are not set-theorists. What is more, there is a multitude of mathematicians (in mainstream non set-theoretic mathemat-

Although I am not going to discuss specifically his philosophical arguments here, the term "plenitudinous Platonism" was introduced by Hartry Field in [4].

* Kobe University Graduate School of System Informatics Rokko-dai 1-1, Nada, Kobe 657-8501

E-mail: fuchino@diamond.kobe-u.ac.jp

The author is supported by Grant-in-Aid for Scientific Research (C) No. 21540150 of the Ministry of Education, Culture, Sports, Science and Technology Japan (MEXT). He would like to thank Andrew Brooke-Taylor and the anonymous referees for their helpful comments. 
ics) who are deeply Platonist in a way quite different from the Platonist stand point set-theorists take, indeed so deeply Platonist that they usually feel no need to express this view explicitly. We shall examine these issues below more in detail.

In Gödel's time, set-theorists could still live with a heliocentric worldview where we had the universe of set theory. In contemporary set theory, however, it became more and more difficult to maintain this view point, in particular, after set-theorists began to deal with the plentitude of models of set theory as a whole which build a rich mathematical construct of higher order. We shall examine a possible extension of classical mathematical Platonism corresponding to this developement.

\section{Pure mathematical Platonism}

First, let us fix the terminology we shall use in the rest of the present article. Since we treat here only Platonism in mathematics, we shall often simply say Platonism to mean mathematical Platonism, or more specifically, such Platonism as is shared by many mathematicians. By mathematicians we mean here not simply the people who would call themselves such, say, in a tax declaration, but rather people who constantly try to establish new mathematical theories by proving theorems.

Concerning the definition of (mathematical) Platonism, Øystein Linnebo [13] defines it in accordance with the definitions widely found in the literature as the conjunction of the theses on

(1) the existence of mathematical objects;

(2) abstractness of the mathematical objects; and

(3) independence of the mathematical objects from intelligent agents and their language, thought, and practices.

However, as far as the Platonism shared by many mathematicians is concerned, one another important thesis seems to be missing here. Namely the stipulation that

(4) there are no other objects than the mathematical in the universe.

For non mathematicians, (4) might seem to be a bit pathologically narrow-minded. However you have to remember that interfaces to the physical world are fully provided in the framework of mathematics by all possible mathematical models available there. Anyway, it should be quite legitimate to consider mathematical Platonism of this form just as much as to consider the purest form of nominalist view according to which only terms and predicates exist. Also, it should be obvious that, for mathematicians (in the sense above), there are no other objects than the mathematical, at least when they are deeply concentrated on the search for mathematical "truth". This feature of mathematics is so obvious for most mathematicians that they usually do not try to formulate their Platonist viewpoint enhanced with (4) unless they are 
forced to do so, for example, when they speak to a general audience, or write an introductory textbooks.

Let us here call this strict form of mathematical Platonism pure mathematical Platonism ${ }^{1}$. That the majority of the mathematicians take this standpoint of pure mathematical Platonism explains also their strong mistrust or even hatred of mathematical logic: if you live thoroughly in a world, you do not feel any need to analyze that world from outside; it is even quite bothersome to have some people in the neighborhood who concern themselves too much with these unnecessarily "philosophical" questions about your world.

But even some logicians, model theorists or researchers of modal logics, for example, can live quite comfortably in this universe of pure mathematical Platonism without any identity crisis, since they can (or rather should) treat their logic(s) as a part of the mathematical universe consisting of sequences of mathematical objects like natural numbers (i.e. not the meta-mathematical natural numbers (or numerals as expressions in meta-mathematics) but the "real" natural numbers as mathematical objects in the universe).

The Platonism of mathematicians I describe here cannot be easily distinguished from working realism (or "conceptual realism" as Paul Bernays [2] put it) in which you merely pretend that you are a Platonist for the sake of efficiency of your mathematical arguments. Above all, the circumstance that they do not usually comment on their Platonist standpoint makes the distinction fairly difficult. But actually, this issue of whether it is a real conviction or merely a pretended one arises whenever we attempt to discuss about Platonist attitude (or indeed any conviction) of somebody.

\section{Platonism with meta-mathematics}

The situation is drastically different for proof theorists: The classical proof theorists are destined to live in a strongly nominalist meta-mathematical world of (either pure or extended) finitary standpoints while modern proof theorists have to live somewhere between this finitary outer world and the "real" world of the Platonist universe because of the more and more impredicative nature of their subjects of research.

The standpoint set-theorists (of set-theory in its most modern form) can take is even more precarious: they are forced to live fully in the universe of pure mathematical Platonism (the set-theoretic universe $V$ ) because of the involved combinatorics they have to deal with. At the same time, they often have to step out from this

\footnotetext{
${ }^{1}$ Surerly the discussions concerning (4) I brought here is too short and incomplete. A thorougher analysis of (4) will be done elsewhere in the future. Here I just want to empahsize that the bold identification of set theoretic universe $V$, which is merely the other name of the class $\{x: x=x\}$, with the mathematical universe in the sense of Platonism obtain certain coherence only under the assumption of (4).
}

$$
-51-
$$


universe to the nominalist outer world of meta-mathematics to discuss relative consistency. They even have to extend and restrict the inverse $V$ from outside to obtain various "models" of set theory via forcing and inner model theory.

In this respect, it is rather amazing that Gödel's standpoint in connection with his contemplation on set theoretic issues was considered as being typically Platonist. However, Gödel stood at the very beginning of the transformation of set theory from the investigation of the single model, the universe $V$ of set theory which the axioms of set theory purport to describe, to the study of the multitude of models constructed by forcing and inner model theory, often in combination with assumptions on the existence of large cardinals. At this early stage, however, the meta-mathematical arguments could still be considered merely as gedankenexperiments of sorts with which we can see more precisely what we can say and what we cannot over the universe of set theory. Even so, this feature of "gedankenexperiments" is something totally missing and, as I mentioned already above, even very disturbing, for the majority of mathematical Platonists.

\section{The set-theoretic multiverse}

Later, as these methods of construction of models of set theory are enough developed, it became more and more difficult to see it as Platonism about the unique universe of mathematics. Rahter we often find ourselves in a situation where we "live" in many different models of set theory simultaneously. Here we live in a parallel world of multiple models of set theory where these models are not just juxtaposed side by side but interwoven in a web of relations: " $M$ is an initial segment of $N$ ", " $M$ is an inner model of $N$ ", " $N$ is a set generic extension of $M$ ", and so forth.

Still, some of these models can be considered to be more natural (or more typical) than others. For example, models with many large cardinals may be seen as more natural compared with other models like $L$ where there cannot be any "large large cardinals"; on the other hand, $L$ should be considered as very typical as the smallest inner model of any model of set theory. Models satisfying strong forcing axioms should also be considered as being natural (e.g. see [7]). Models satisfying the Maximality Principle [10] or the Inner Model Hypothesis [5] should be also considered to be natural; philosophical discussions about the latter can be also found in [1]. $\operatorname{PFA}(S)[S]$ and its strengthenings (see, e.g. [15]) of Todorčević seem to be quite artificial but strong consequences we obtain in these models suggest that there might be something more intrinsic hidden in these models.

However, there is no guarantee that all of these natural properties of models converge to one single model of set theory. For example, Hugh Woodin, recently discovered two natural arguments in terms of his $\Omega$-logic, one of which concludes $2^{\aleph_{0}}=\aleph_{2}$ while the other implies $\mathrm{CH}$. 
With this background, we see that a new extended mathematical Platonism is needed in which multiplicity of models of set theory as a whole is seen as the unique mathematical "multiverse" and even the meta-mathematics attached to them should also be included in this Platonist picture. Conventional mathematics done inside ZFC can be embedded in such a Platonist multiverse naturally by considering such mathematics to be performed simultaneously in all of the models of ZFC in the multiverse.

It seems to me that this sort of multiverse view is the only feasible way out of the identity crisis of modern set-theorists if they wish to retain their Platonist view of mathematics as much as possible. In [6], we showed that a formalism naturally corresponding to this extended Platonism can be obtained as a conservative extension of the theory of the single universe of set theory we choose as the base point (e.g. ZFC or ZFC plus some additional axioms) ${ }^{2}$.

From the point of view of the working realism of mathematicians, it only remains to be seen whether such a multiverse version of the Platonism leads us to deeper knowledge of mathematical infinity.

\section{References}

[1] Tatiana Arrigoni and Sy D. Friedman, Foundational implications of the Inner Model Hypothesis, to appear in Annals of Pure and Applied Logic.

[2] Paul Bernays, "Sur le platonisme dans les mathématiques", L'Enseignement Mathématique, Vol.34 (1935), 52-69.

[3] Mark Balaguer, Platonism and anti-Platonism in mathematics, Oxford University Press, (1998).

[4] Hartry Field, Which undecidable mathematical sentences have determinate truth values?, in: H. Garth Dales and Gianluigi Oliveri (eds.), Truth in Mathematics, Oxford University Press (1998), 291-310.

[5] Sy D. Friedman, Internal consistency and the inner model hypothesis, Bulletin of Symbolic Logic, 12(4), (2006), 591-600.

[6] Sy D. Friedman, Sakaé Fuchino and Hiroshi Sakai, "On the set-generic multiverse", preprint.

[7] Sakaé Fuchino, "Forcing Axioms and the Continuum Problem", Sugaku Expositions, Vol.21, No.2 (2008), 147-159.

[8] Kurt Gödel, "What is Cantor's Continuum Hypothesis?" (1964), in: Benacerraf and Putnam (eds.), "Philosophy of Mathematics: Selected Readings, Cambridge", Cambridge University Press. Second edition (1983).

[9] Kurt Gödel, "Some basic theorems on the foundations of mathematics and their implications", in Collected Words, S. Feferman et al, ed., Oxford: Oxford University Press,

${ }^{2}$ There are now many discussions about "plenitudinous Platonism" and more specifically on the set-theoretic multiverse view. Readers may consult, [3], [10], [11], [12] among others. 
vol. III (1995), 304-323.

[10] Joel D. Hamkins, The set-theoretical multiverse: a natural context for set theory, Annals of the Japan Association for Philosophy of Science, special issue Mathematical Logic and Its Applications, Vol.19 (2011), 37--55.

[11] Joel D. Hamkins, The set-theroretic multiverse, to appear in Review of Symbolic Logic. (arXiv:1108.4223v1 [math.LO])

[12] Peter Koellner, Truth in Mathematics: The Question of Pluralism, in: Otávio Bueno and Øystein Linnebo (eds.), New Waves in Philosophy of Mathematics, Palgrave Macmillan (2009), 80-116.

[13] Øystein Linnebo, "Platonism in the Philosophy of Mathematics", The Stanford Encyclopedia of Philosophy (Fall 2011 Edition), Edward N. Zalta (ed.), http://plato.stanford.edu/archives/fall2011/entries/platonism-mathematics/.

[14] Donald A. Martin, "Multiple universes of sets and indeterminate truth values", Topoi, 20(1) (2001), 5-16.

[15] Franklin D. Tall, $\mathrm{PFA}(S)[S]$ : more mutually consistent topological consequences of PFA and $V=L$ Canadian Journal of Mathematics, to appear.

[16] "Philosophy of mathematics", Wikipedia: The Free Encyclopedia, Wikimedia Foundation Inc., 6 December 2011 Web. 30 December (2011).

http://en.wikipedia.org/wiki/Philosophy_of_mathematics

(Received 2012.1.20; Accepted 2012.2.7) 